\title{
Inelastic Electroproduction of Charmonium at HERA
}

\author{
M. Steder
}

DESY, Notkestr. 85, 22607 Hamburg, Germany

\begin{abstract}
Inelastic electroproduction of $J / \psi$ mesons is studied in $e p$-scattering at HERA in the range $3.6<Q^{2}<100 \mathrm{GeV}^{2}$. The data were taken with the $\mathrm{H} 1$ detector in the years 2004 through 2006 and correspond to an integrated luminosity of $\mathcal{L} \approx 258 \mathrm{pb}^{-1}$. Single differential and double differential cross sections are measured with increased precision compared with previous analyses.
\end{abstract}

\section{Introduction}

Inelastic $J / \psi$ production in $e p$ collisions is dominated by the process of photon-gluon fusion where a photon from the incoming electron and a gluon from the proton couple to a $c \bar{c}$ pair. The process can be calculated within non-relativistic QCD (NRQCD) where the cross section is a sum over all possible intermediate $c \bar{c}$ states, colour singlet (CS) and colour octet $(\mathrm{CO})$ states. The amplitude for each $c \bar{c}$ state with definite colour and angular momentum factorises into a short distance term which can be calculated in NRQCD and a long distance matrix element (LDME) describing the transition to a $J / \psi$ meson. The LDMEs are not calculable and have been determined from $J / \psi$ production data in $p \bar{p}$ collisions where the $\mathrm{CO}$ contributions were found to be sizable. Previous HERA measurements in photoproduction showed good agreement with NLO calculations of the Colour Singlet Model (CSM), which takes only colour singlet states into account. But small colour octet contributions could not be excluded.

In this talk a measurement of single differential and double differential cross sections of inelastic $J / \psi$ in electroproduction is presented which provides improved statistical precision in comparison to the previous measurements. The kinematic range ist restricted to $3.6<$ $Q^{2}<100 \mathrm{GeV}^{2}, 50<W_{\gamma p}<225 \mathrm{GeV}$ for medium elasticities $0.3<z<0.9$ and the squared transverse momentum of the $J / \psi$ meson in the photon-proton center of mass system $p_{t}^{* 2}>1 \mathrm{GeV}^{2}$. The systematic error has been determined to be $10-11 \%$.

The data are compared with predictions from two Monte Carlo models generating events according to the Color Singlet model in leading order, CASCADE [3] and EPJPSI [4]. In CASCADE, higher order parton emissions based on the CCFM [5] evolution equations are matched to $\mathcal{O}\left(\alpha_{s}\right)$ matrix elements in which the incoming parton can be off-shell. The Monte Carlo generator EPJPSI [4] is based on the DGLAP evolution [6], assuming collinear factorization of the parton density distributions and the hard matrix elements.

\section{Cross Section Measurement}

Differential cross sections are determined separately for the decay channels into muons and into electrons. The cross sections are then combined by error weighted averaging. The production cross section is determined by measuring the number of events, correcting for detector efficiencies and acceptances and dividing by the integrated luminosity of $258 \mathrm{pb}^{-1}$ and the corresponding branching ratio of $\mathcal{B R}(J / \psi \rightarrow e e)=(5.94 \pm 0.06) \%$ and $\mathcal{B R}(J / \psi \rightarrow$ $\mu \mu)=(5.93 \pm 0.06) \%$, respectively $[7]$. 

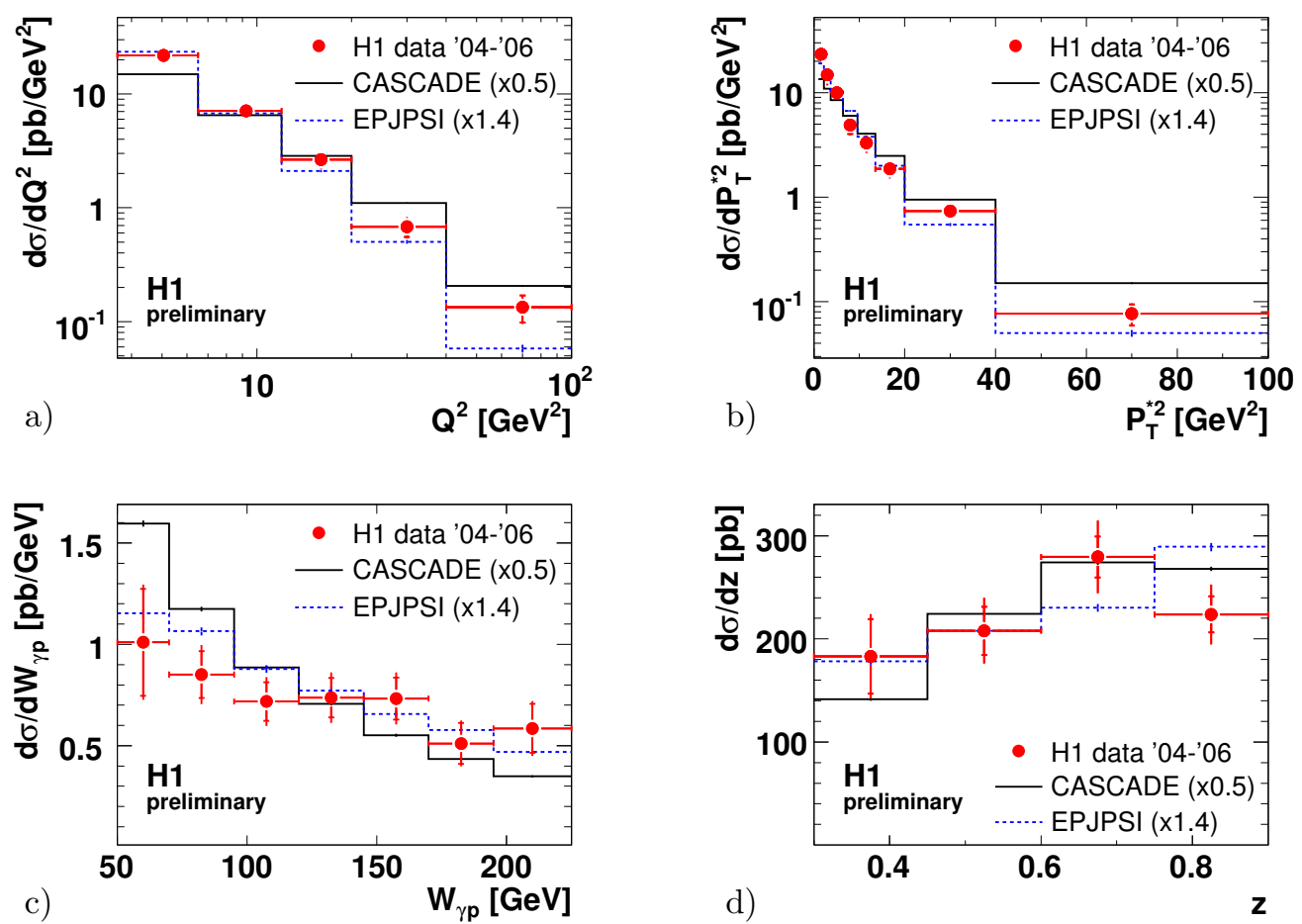

Figure 1: Differential $J / \psi$ cross sections for the kinematic range $Q^{2}>3.6 \mathrm{GeV}^{2}, 50<$ $W_{\gamma p}<225 \mathrm{GeV}, 0.3<z<0.9$ and $p_{t, \psi}^{* 2}>1 \mathrm{GeV}$, as functions of a) $Q^{2}$, b) $p_{t, \psi}^{* 2}$, c) $W_{\gamma p}$, d) $z$. The data are compared to the predictions from CASCADE (solid line) and EPJPSI (dashed line).

The measured cross sections are not corrected for contributions from secondary $J / \psi$ mesons from the decays of $B$-Mesons, $\chi_{c}$ or $\psi(2 S)$ mesons. The fraction of events arising from diffractive $\psi(2 S)$ meson production with subsequent decay of the $\psi(2 S)$ into $J / \psi+X$ is estimated to be $1.5 \%$ in the total sample and about $5 \%$ in the highest $z$ bin. The fraction of events arising from $B$ mesons decaying into $J / \psi+X$ is estimated to be $3.6 \%$ in the total sample and about $20 \%$ in the lowest $z$ bin.

Differential cross sections are measured as functions of the following variables: $Q^{2}, W_{\gamma p}$, $z$, the transverse momentum squared, $p_{t, \psi}^{* 2}$, of the $J / \psi$ in the $\gamma^{*} p$ center of mass frame. The measured differential cross sections are shown in Figure 1. Shape comparisons of the data are performed with predictions from the CASCADE generator (solid line) and EPJPSI (dashed line), The total cross sections of the Monte Carlo simulations are normalized to the total cross section of the measurement. CASCADE shows a reasonable description of the dependence on $Q^{2}$. It has a somewhat harder distribution in $p_{t, \psi}^{* 2}$ than the data. In contrast EPJPSI has a much steeper distribution in $Q^{2}$ and in $p_{t, \psi}^{* 2}$. Both programs predict a somewhat flatter distribution of the cross section in $z$.

The distributions in $p_{t, \psi}^{* 2}$ and $z$ are further investigated in Figures 2 and 3 by dividing 

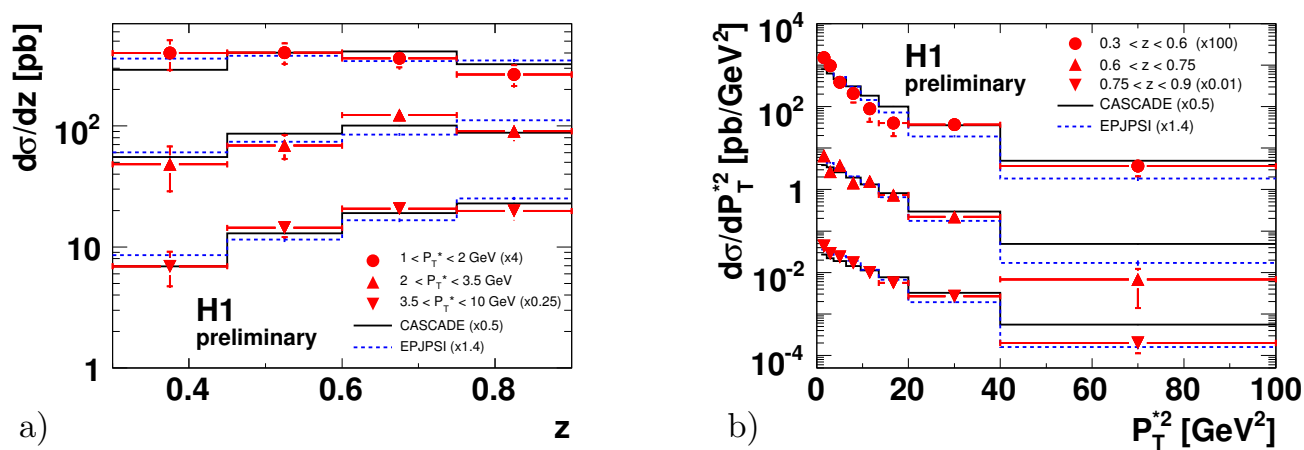

Figure 2: Differential $J / \psi$ cross sections, $d \sigma / d z$ as a function of a) $z$ in three bins of $p_{t, \psi}^{*}$ and b) $d \sigma / d p_{t, \psi}^{* 2}$ as a function of $p_{t, \psi}^{* 2}$ in three bins of $z$. The predictions from CASCADE (solid line) and EPJPSI (dashed line) are also shown.

the sample into bins of $z$ and $p_{t, \psi}^{*}$, respectively. Both Monte Carlo models describe the $z$ distribution in the largest bin of $p_{t, \psi}^{*}$ and the dependence on $z$ of the slope of $p_{t, \psi}^{* 2}$. At small values of $z$, the dependence on $p_{t, \psi}^{* 2}$ is better described by CASCADE while EPJPSI agrees better at large values of $z$.

\section{Conclusions}

A measurement of inelastic $J / \psi$ production is performed with improved statistics and systematics with respect to previous measurements. Differential and double differential cross sections are measured and compared with predictions from the MC models CASCADE and EPJPSI. Both models are not capable of describing all aspects of the data. No indications for large NRQCD contributions are seen.

\section{References}

[1] Slides: http: //indico. cern. ch/contributionDisplay . py? contribId=78\&sessionId=13\&conf Id $=9499$

[2] C. Adloff et al. [H1 Collaboration], Eur. Phys. J. C 25 (2002) 41 [hep-ex/0205065].

[3] H. Jung and G.P. Salam, Eur. Phys. J. C 19 (2001) 351 [hep-ph/0012143]; H. Jung, Comput. Phys. Commun. 143 (2002) 100 [hep-ph/0109102].

[4] H. Jung, in: Proc. of the Workshop on Physics at HERA, ed. W. Buchmüller and G. Ingelman, Hamburg (1992), Vol. 3, p. 1488; H. Jung, D. Krücker, C. Greub and D. Wyler, Z. Phys. C 60 (1993) 721.

[5] M. Ciafaloni, Nucl. Phys. B 296 (1988) 49; S. Catani, F. Fiorani and G. Marchesini, Phys. Lett. B 234 (1990) 339; idem, Nucl. Phys. B 336 (1990) 18; G. Marchesini, Nucl. Phys. B 445 (1995) 49.

[6] V.N. Gribov and L.N. Lipatov, Yad. Fiz. 15 (1972) 781 [Sov. J. Nucl. Phys. 15 (1972) 438]; G. Altarelli and G. Parisi, Nucl. Phys. B 126 (1977) 298; Y.L. Dokshitzer, Sov. Phys. JETP 46 (1977) 641 [Zh. Eksp. Teor. Fiz. 73 (1977) 1216].

[7] W.-M. Yao et al. [Particle Data Group] J. Phys. G 33, 1 (2006). 

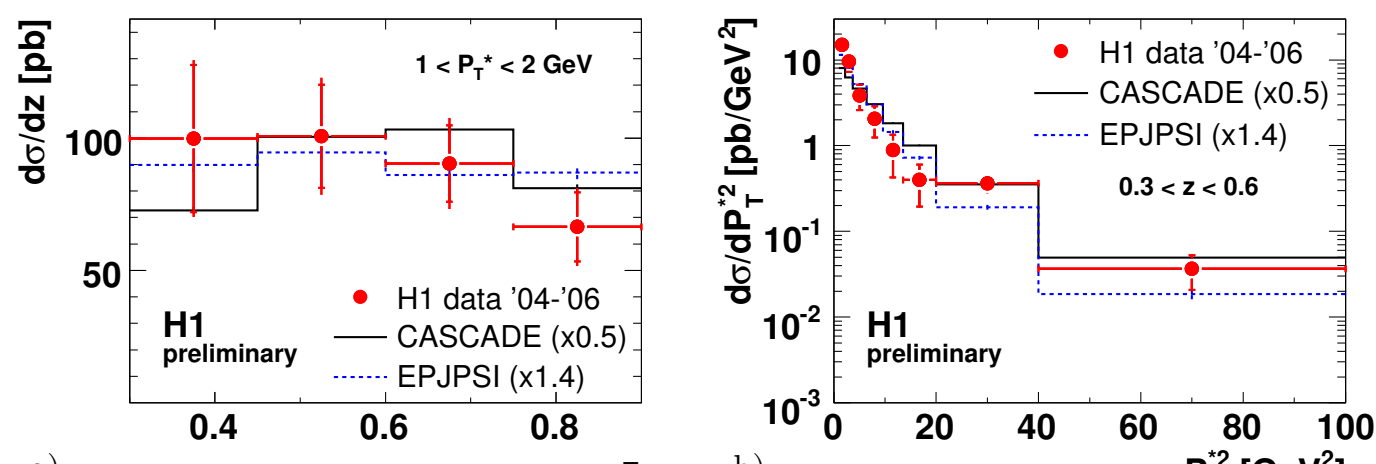

a)

$\mathbf{Z}$

b) $\mathbf{P}_{\mathrm{T}}^{* 2}\left[\mathrm{GeV}^{2}\right]$
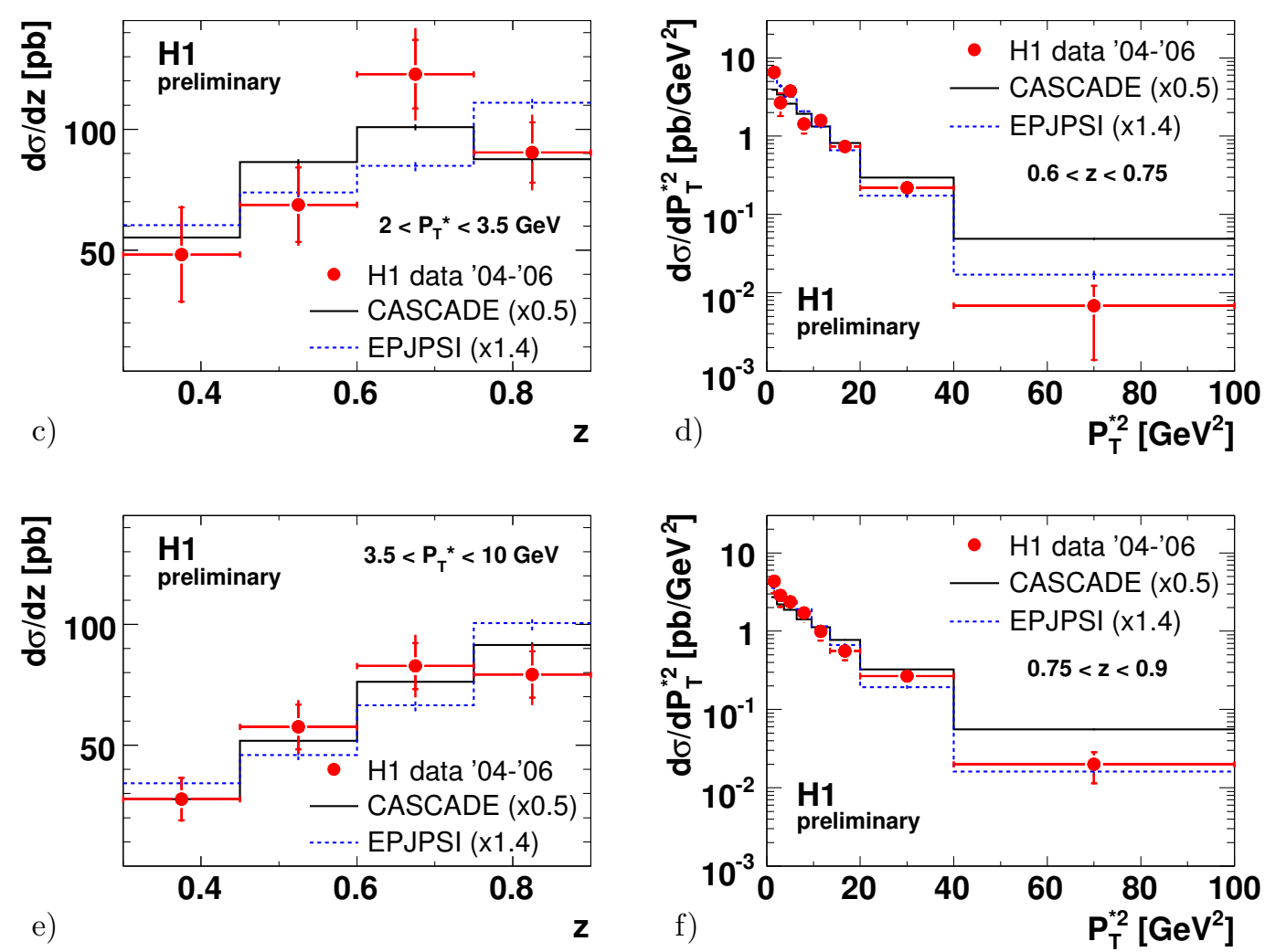

Figure 3: Differential $J / \psi$ cross sections, $d \sigma / d z$ as a function of $z$ in three bins of $p_{t, \psi}^{*}$, a) $1<p_{t, \psi}^{*}<2 \mathrm{GeV}$, c) $2<p_{t, \psi}^{*}<3.5 \mathrm{GeV}$, e) $3.5<p_{t, \psi}^{*}<10 \mathrm{GeV}$ and $d \sigma / d p_{t, \psi}^{* 2}$ as a function of $p_{t, \psi}^{* 2}$ in three bins of $z$, b) $0.3<z<0.6$, d) $0.6<z<0.75$ and f) $0.75<z<0.9$. The predictions from CASCADE (solid line) and EPJPSI (dashed line) are also shown. 